\title{
Induced Polarization Modelling Using the Fractal Model to Complex Resistivity for Environmental Application
}

\author{
V. J. da C. Farias* and B. R. P. da Rocha \\ Universidade Federal do Pará, Programa de Pós Graduação em Engenharia Elétrica, Ruas Augusto Corrêa 01, Belém-Pará-Brazil
}

\begin{abstract}
A finite element code to simulate the forward and inverse induced polarization response to a 2-D model was developed. The "Fractal Model to Complex Resistivity" was applied as an intrinsic electrical property of the medium. The simulations were carried out in five different frequencies. The inversion of the complex resistivity parameter was applied to each frequency, further it was applied also to each cell of the finite element mesh in order to analyze the possibility of using the fractal model parameters in the qualitative and quantitative interpretation of the induced polarization response to this geological geometry. The results showed that the anomalies are well detected by the image of the fractal model parameters.
\end{abstract}

Keywords: complex resistivity, finite element, forward modeling, fractal, inverse modelling

\section{Introduction}

The induced polarization phenomena (IP) have an electrochemical origin and are frequently used to explain the electrical response of geological layers or strucutures (Rocha and Habashy, 1995a, b) and biological environments (Rocha et al., 1997). As a consequence of these phenomena, the electrical resistivity (or their reciprocal electrical conductivities) are a frequency-dependent complex variable. The method of induced polarization in a geophysical environment uses the complex character of the conductivity of rocks in lowfrequencies to perform a variety of prospecting activities. The method was originally developed to search for disseminated ores. The development of this technique made possible to apply the IP method for mineral discrimination (Sampaio et al., 1998) and environmental investigation (Kemna et al., 1999, 2000).

A quantitative interpretation of induced polarization data in geophysical prospecting is difficult due mostly to the fractal nature of geologic structures. To perform this interpretation, it is necessary a physical model to explain the behavior of the polarizable medium for a large frequency range of the electromagnetic spectrum.

Rocha (1995) introduced a physical model that considers the fractal effects of the porous surface and includes the bulk response of rocks at low frequencies of electromagnetic source. The introduction of the fractal roughness factor, permits the investigation of the texture of rocks, which is a very important parameter to explain their electrical properties.

\footnotetext{
* Corresponding author: valcir@ufpa.br
}

Rocha and Habashy (1995a) applied the fractal model of complex resistivity as an intrinsic electrical properties of a horizontally stratified medium (1-D model) and analyzed its IP response. They observed that the fractal parameter of the fractal model dominates the phase response of the apparent resistivity.

In this paper shaped bodies were simulated (2-D geological model) using finite element mesh and the fractal model was introduced as the complex resistivity associated to the intrinsic electrical property of this medium. This simulation was carried out in five different frequencies. At each frequency the inversion of the complex resistivity was obtained. Furthermore, for each cell of the finite element mesh, the fractal model parameters inversion was undertaken to analyze the possibility of using the fractal model parameters, for qualitative and quantitative interpretations of the induced polarization response of this geological geometry. In this model the fractal parameter $(\eta)$ represents the fractal index, which can be related to the rock texture.

\section{The Fractal Model}

The fractal model for complex resistivity of rocks was first introduced by Rocha and Habashy (1995a) using an analog model circuit which included the diffusity $(\mathrm{K})$ of ions in the vicinity of the electrode/electrolyte interface, and a fractal parameter $(\eta)$. In addition, Rocha (1995) proposed the adoption of a fractal time to substitute the diffusivity of ions.

Rocha (1995) considered that, throughout the whole frequency spectrum, two main paths could electrically characterize rocks: one representing the free porous channel, and another representing the blocked porous channel. The free chan- 
nel represents the electrical paths in the rock that are free of clay or metallic particles, while the blocked channels represent the paths which encounter these minerals.

The model includes a fractal rough surface impedance element. This element will be responsible for the behavior of the material at very low frequency signals. The electrically charged double layer in the porous medium may be represented by a pure resistive term (resulting from loss of energy due the collision of the free carriers during their motion across the charged double layer) and capacitive term (caused by oscillations of the bound charges in the double layer). This couple of parameters will respond by the medium frequency response of rock.

The capacitance associated with the bulk polarization of rocks is represented by their capacitive path in parallel with DC resistivity of rock. This capacitance in combination with the bulk resistivity $\left(\rho_{0}\right)$ of the naterial will be responsible for the very high frequency response. In the absence of this capacitive element, the dielectric constant will have no upper bound at high frequencies.

Representing the time dependence of the electric field as $e^{i \omega t}$, the expression proposed by Rocha (1995) for the complex electrical resistivity $\rho(\omega)$ is given by:

$$
\rho(\omega)=\rho_{o}\left[1-m\left(1-\frac{1}{1+\frac{1+u}{\delta_{r}(1+v)}}\right)\right] \gamma_{h}
$$

where $\rho_{o}$ is the DC resistivity of the material; $m$ is the chargeability; $\delta_{r}$ is the grain percent resistivity; $\gamma h=1 /\left(1+i \omega \tau_{o}\right) ; u$ $=i \omega \tau(1+v) ; \mathrm{v}=\left(i \omega \tau_{f}\right)^{-\eta} ; \tau$ is relaxation time constant related the double layer oscillations; $\tau_{o}$ is the sample relaxation time constant; $\tau_{f}$ is the fractal relaxation time and it is related to the time involved in the transference of charge and energy in the rough interfaces; and $\eta$ is the parameter directly related to the fractal geometry of the medium.

Olhoeft (1985) measured the complex conductivity of different kinds of rocks, including uncontaminated soils and soils contaminated with chemical products and waste materials. By means of the least squares fit, the intrinsic fractal model parameters were estimated by (Rocha, 1995) from experimental data of Olhoeft (1985). Table 1 presents the intrinsic fractal parameters obtained for some Olhoef data.

\section{Two-Dimensional Forward Modelling}

The aim of the forward modeling in geophysical prospecting with electrical method is to calculate the apparent resistivity, for the electrode configuration adopted, and a given subsurface conductivity structure. We assumed in this study that the region of interest might be represented as a 2-D complex resistivity distribution. Neglecting the electromagnetic induction effects, the forward problem is defined by
Poison's equation for a point source with real current I, thus:

$-\nabla\left(\frac{1}{\rho^{*}(x, z)} \nabla V(x, y, z)\right)=I \delta(x) \delta(y) \delta(z)$

where $V(x, y, z)$ is the potential and $\rho^{*}(x, z)$ is the complex resistivity of the medium which is given by (1).

The resistivity distribution $\rho^{*}(x, z)$ has two dimensions. However, the current electrode is a point source (3D problem). By taking the Fourier transform of the equation above in the $y$-direction, the $3 \mathrm{D}$ potential distribution $V(x, y, z)$ is reduced to a $2 \mathrm{D}$ transformed potential, thus:

$-\nabla\left(\frac{l}{\rho^{*}} \nabla V^{*}(x, \lambda, z)\right)+\lambda^{2} \frac{V^{*}(x, \lambda, z)}{\rho^{*}}=I \delta(x) \delta(z)$

where $V^{*}$ is the transformed complex potential, $l$ is the Fourier transformation variable.

The Equation (2) was solved using the finite element method for an appropriated boundary condition. The finite element discretization of the Equation (2) gives rise to the system of algebraic equations:

$A V^{*}=b$

where the conductance matrix $A$ is a $N \times N$ symmetric sparse banded matrix, with $N$ been the number of nodes; $b$ is the current vector. To reduce the memory space necessary to store the conductance matrix, we stored the matrix by diagonal.

Table 1. Fractal Model Parameters Obtained by Rocha (1995) to the Experimental Data of Olhoeft (1985).

\begin{tabular}{llllllll}
\hline Sample & $\begin{array}{l}\rho_{\mathrm{o}} \\
(\Omega . \mathrm{m})\end{array}$ & $\mathrm{m}$ & $\delta_{\mathrm{r}}$ & $\begin{array}{l}\tau \\
(\mu \mathrm{s})\end{array}$ & $\begin{array}{l}\tau_{\mathrm{f}} \\
(\mathrm{ms})\end{array}$ & $\eta$ & $\begin{array}{l}\tau_{\mathrm{o}} \\
(\mathrm{ps})\end{array}$ \\
\hline Oh-1 & 400.0 & .439 & .303 & .342 & 97.8 & .323 & .005 \\
Oh-2 & 30.0 & .608 & .617 & .207 & 29900 & .238 & .026 \\
Oh-3 & 30.0 & .691 & .292 & .255 & 13935 & .288 & .024 \\
Oh-4 & 7.877 & .756 & 2.042 & .121 & .783 & .378 & .009 \\
Oh-5 & 5.669 & .995 & 4.046 & .157 & .001 & .159 & .294 \\
\hline
\end{tabular}

Note: Oh-1 = Tuff with its natural content of water; Oh-2 = Uncontaminated smectite soils; Oh-3 = Organic waste contaminated smectite soils; Oh-4 = Uncontaminated montmorillonite soils; Oh-5 = Organic waste contaminated montmorillonite soils.

The complex potential $V^{*}(x, \lambda, z)$ obtained in different wavernumbers (generally five to ten) are used to reconstruct the potential $V(x, y, z)$ by an inverse Fourier Transform. When the potential electrodes are located in the plane $y=0$, the inverse Fourier transform reduces to the integral:

$V^{*}(x, 0, z)=\frac{1}{\pi} \int_{0}^{\infty} V^{*}(x, \lambda, z) d \lambda$ 
The complex apparent resistivity was calculated with the expression:

$$
\rho_{a}=K \frac{V}{I}
$$

where $K$ is the geometric factor related to the electrode configuration.

\section{Inverse Modelling}

To recover the instrinsic electrical proprieties of the media its necessary to solve an inverse problem. The objective function being minimized in the inversion is of the same kind as the used by (Xia et al., 1994) and is given by expression:

$C(\bar{x})=\frac{1}{2}\left\{\mu\left[\left\|\overline{\bar{W}}_{d}\left(f(m)-d^{o b s}\right)\right\|^{2}-\chi^{2}\right]+\left\|\overline{\bar{W}}_{x}\left(m-m_{r}\right)\right\|^{2}\right\}$

where $\mu(0<\mu<\infty)$ is the regularization parameters (Lagrange multiplier); $\chi^{2}$ is the prescribe value of data misfit; $f(m)$ is the operator of the forward solution; $d^{\text {obs }}$ is the measured data vector, usually corrupted with some level of noise; $m r$ is the reference model which includes all a priori information on the complex resistivity; $W_{x}$ is the inverse of the model covariance matrix; and $W_{d}$ is the inverse of the data covariance matrix. If the measured noise is stationary and uncorrelated, then $W_{d}=\operatorname{diag}\left(1 / \sigma_{j}^{2}\right)$ where $\sigma_{j}$ is the rms deviation of the noise for the jth measurement.

To solve the above optimization problem, we employed the iterative Gauss-Newton approach, where each step $j$ of the complex linear system is solved by an updating $\Delta m j$ value.

$$
\begin{aligned}
& {\left[J^{H} \cdot W_{d}^{T} \cdot W_{d} J^{H}+W_{x}^{T} \cdot W_{x}\right] \cdot \Delta m_{j}=} \\
& J^{H} \cdot W_{d}^{T} \cdot W_{d} \cdot\left[d^{o b s}-f\left(m_{j}\right)\right]-W_{x}^{T} \cdot W_{x} \cdot m_{j}
\end{aligned}
$$

where $J$ is the sensitivity matrix (Jacobian matrix). We solve the linear system above using the gauss elimination method. The same methodology it was used to recovered the parameters of the fractal model.

\section{Results}

The 2-D model was divided into $147 \times 20$ cells $(2940$ cells), the region of interest has considered only $76 \times 10$ cells (760 cells). The measured data were generated using a dipole-dipole array with a dipole length of $2 \mathrm{~m}$, and the electrode configuration consists in introducing a current into the medium through a pair of electrodes and the voltage was measured in another pair of electrodes. The geometric factor of this electrode configuration is presented in Equation (9):

$$
K=\frac{1}{A M}+\frac{1}{B N}-\frac{1}{A N}-\frac{1}{B M}
$$

where $\mathrm{AM}, \mathrm{AN}, \mathrm{BM}$ e $\mathrm{BN}$ are the distances between the current (A and $\mathrm{B}$ ) and potential electrodes ( $\mathrm{M}$ and $\mathrm{N})$.

The data were collected using 20 electrodes with 8 $n$-spacing and the results are represented in the form of pseudo-sections. The simulations were performed in five different frequencies sampled logarithmically in an interval of $0.1 \mathrm{~Hz}$ to $64 \mathrm{~Hz}$. The data were contaminated with gaussian random noise with a standard deviation of $5 \%$ of the datum value. The inverse model was obtained in the five different frequencies, and there was two synthetic models considered in these simulations: Models 1 and 2.

\subsection{Model 1}

Model 1 (Figure 1) consists of one block intruded in the half-space. The fractal model parameters of the half-space are the same of the sample Oh-4 (Table 1). The block was considered with the fractal model parameters of the sample Oh-5.

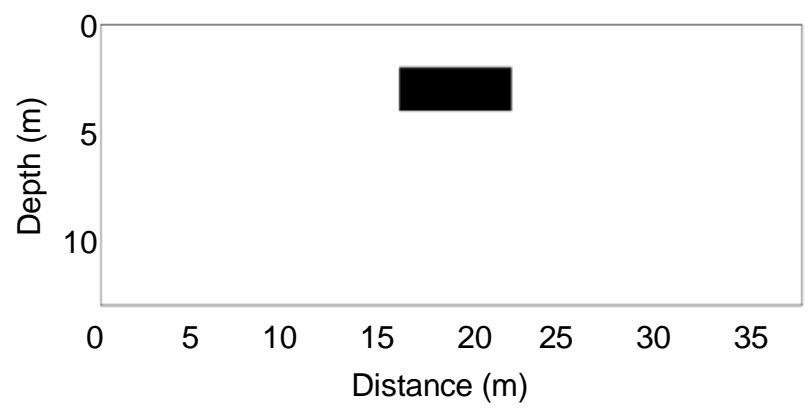

Figure 1. Model 1 consists of a block embedded in the half-space (the dimension of the block is $6 \times 2$ ).

The pseudo section (apparent resistivity) of the forward modeling for the frequencies from 0.1 to $64 \mathrm{~Hz}$ is shown in Figure 2. The influence of the block can be detected only in the phase angle of the apparent complex resistivity response. Figure 3 presents the apparent fractal model parameters $\eta, \delta_{r}$, $m$ and $\tau_{f}$ recovered from the apparent complex resistivity in all frequencies. The apparent fractal parameters had the same behavior of the phase angle of the apparent complex resistivity.

The amplitude and phase angle of the inverse model for frequencies from 0.1 to $64 \mathrm{~Hz}$ (the minimum and the maximum values for the frequency range, respectively) are shown in Figure 4. The contamination of the half-space (the block) can be observed, and it is discretely detected by the phase angle response values, but the shape of the body was not very well defined, mainly when it was analyzed in the lowest frequency signals.

The image of the distribution of the intrinsic fractal model parameters recovered from the inverse model for all frequencies is shown in Figure 5. The image of the parameters $\eta$ and $\delta_{r}$, Figure 5(a) and (b), respectively, shows clearly the 

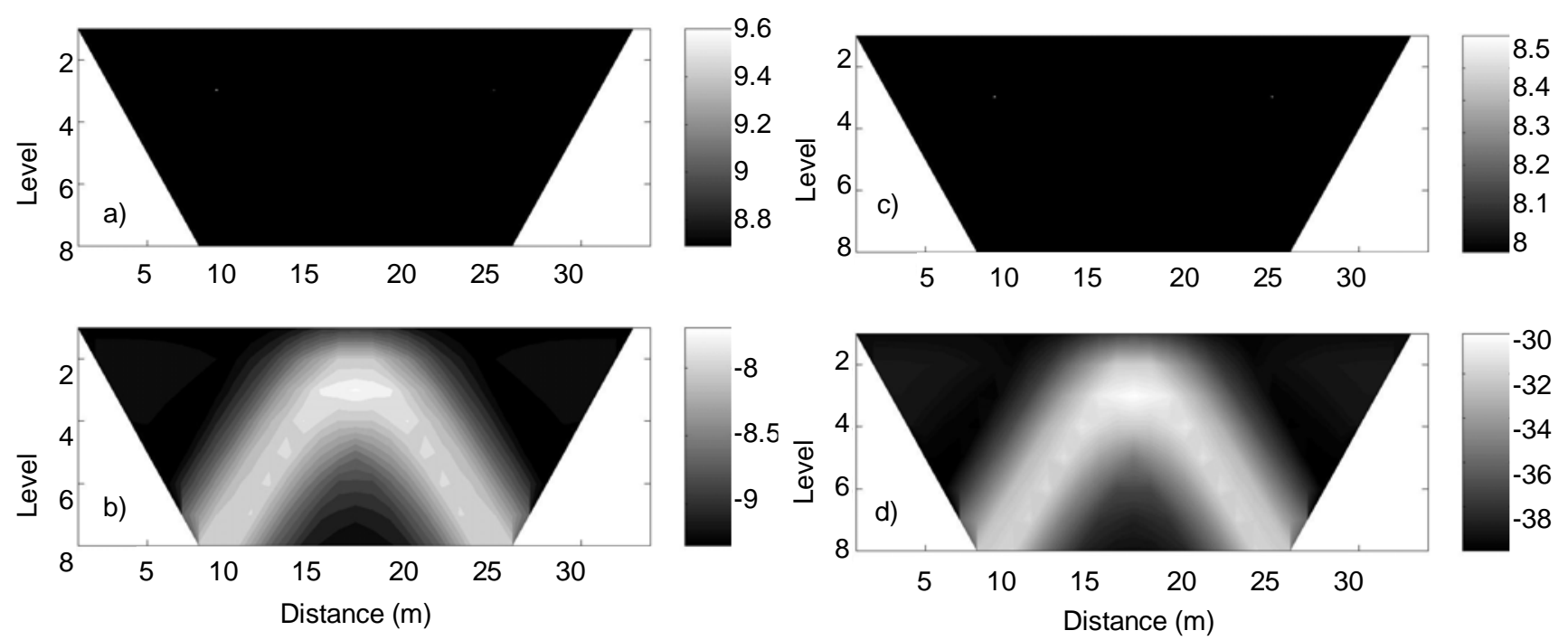

Figure 2. Apparent complex resistivity Pseudosection in frequency of 0.1 and 64Hz: (a) amplitude $(\Omega . \mathrm{m})$ in $0.1 \mathrm{~Hz}$; (b) phase angle (mrad) in $0.1 \mathrm{~Hz}$; (c) amplitude $(\Omega . \mathrm{m})$ in $64 \mathrm{~Hz}$; (d) phase angle (mrad) in $64 \mathrm{~Hz}$.
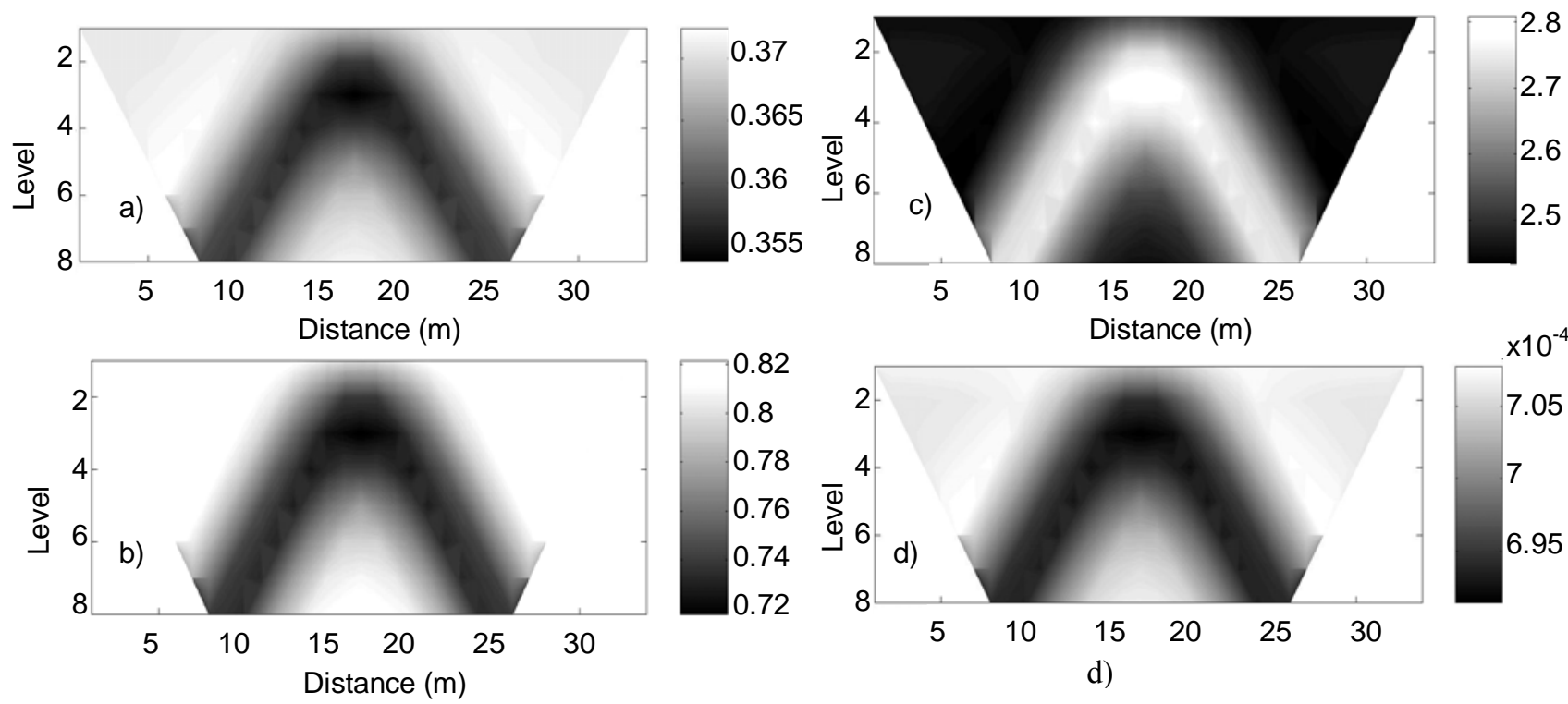

Figure 3. Apparent fractal parameter pseudo section recovered from the apparent complex resistivity pseudo section: (a) fractal parameter $(\eta)$; (b) parameter $(\delta \mathrm{r})$; (c) Chargeability $(\mathrm{m})$; (d) fractal relaxation time $(\tau \mathrm{f})$. 

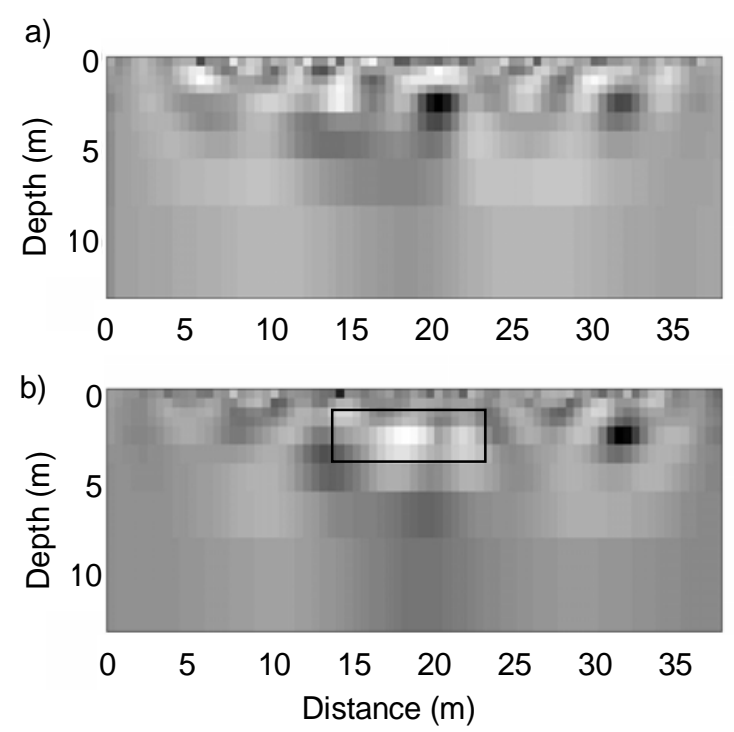
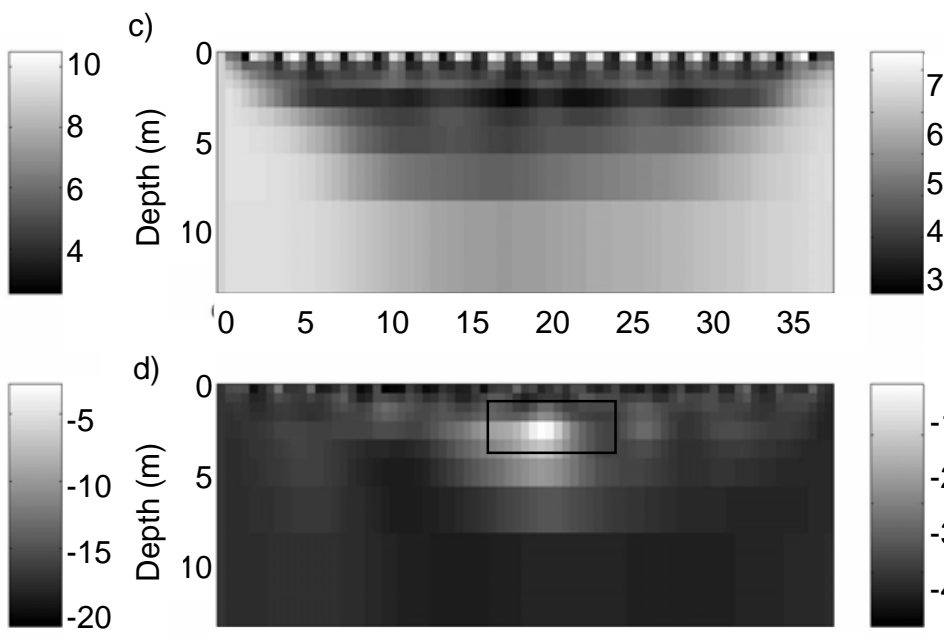

d)

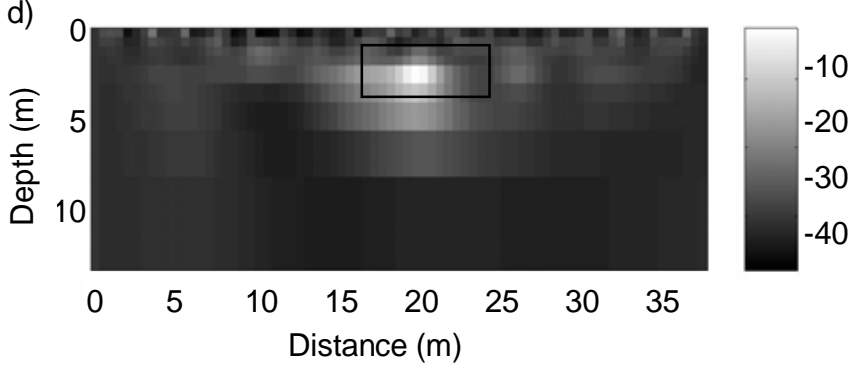

Figure 4. Inverse model of the model 1: (a) and (b) amplitude and phase angle in the Frequency $0.1 \mathrm{~Hz}$, respectively; (c) and d) amplitude and phase angle in the frequency $64 \mathrm{~Hz}$.
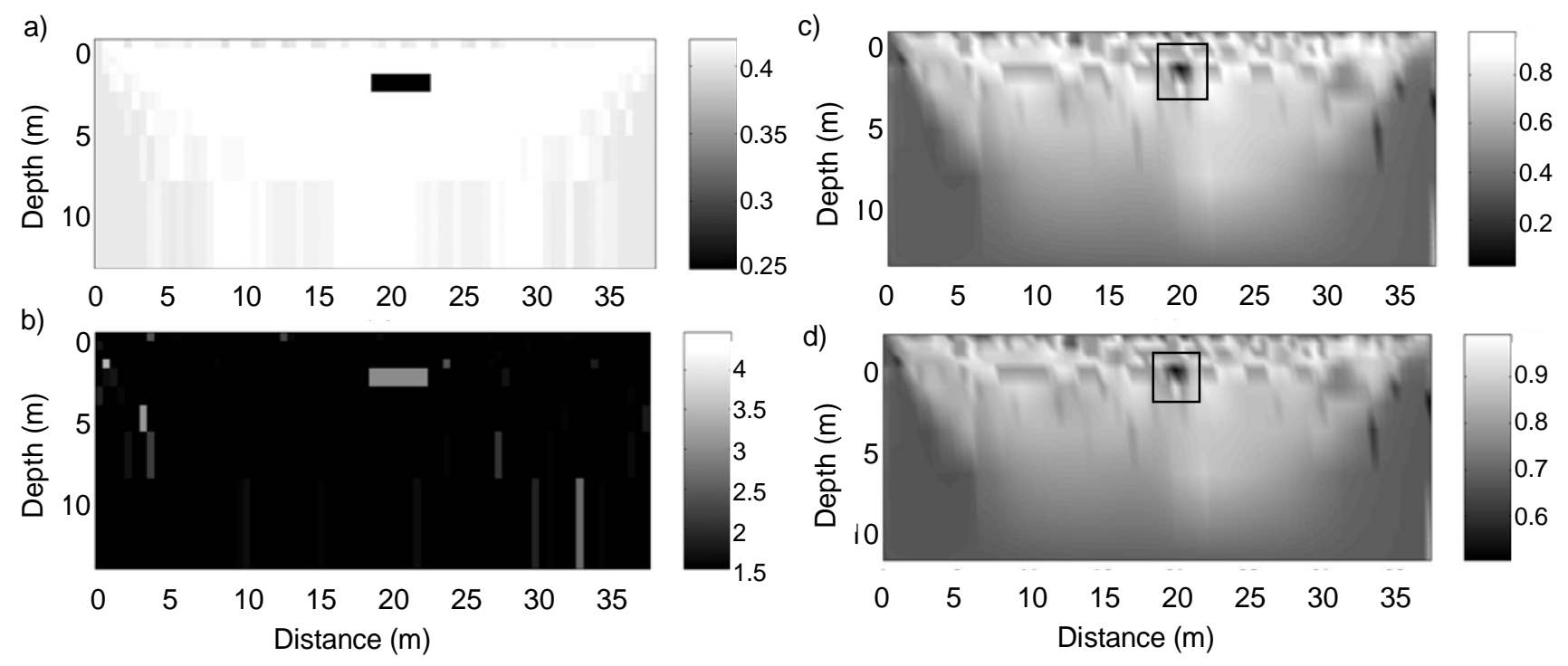

Figure 5. Image of the intrinsic fractal model parameters to model 1: (a) fractal parameter $(\eta)$; (b) parameter $\left(\delta_{\mathrm{r}}\right)$; (c) chargeability $(\mathrm{m})$; (d) fractal relaxation time parameter $\left(\tau_{\mathrm{f}}\right)$. 
position and the form of the block (the contamination introduced in the experiment). The image of the chargeability $(m)$ and fractal relaxation time $\left(\tau_{f}\right)$ parameters (Figure 5 (c) and (d), respectively) cannot be used for a precise definition of the body position.

\subsection{Model 2}

Model 2 shown in Figure 6 consists of one block buried in the second layer of two layered medium. The parameters of the model of the first and second layer were the same of the samples Oh-1 and Oh-2, respectively; the block parameters were the same of the sample Oh-3. All these values of the samples of Oh-1, Oh-2 and Oh-3 were summarized in Table 1.

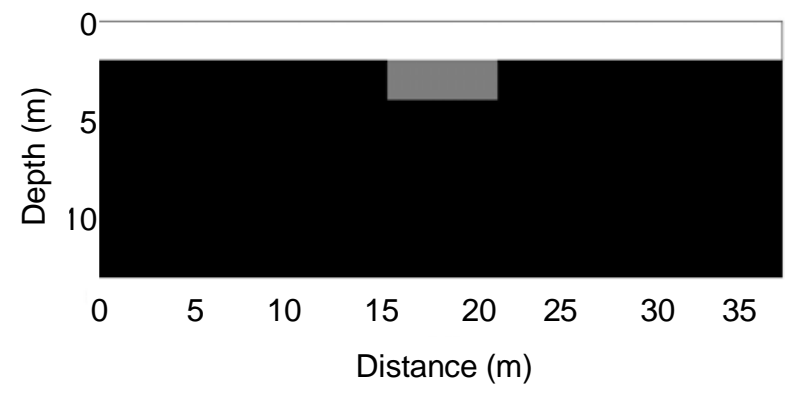

Figure 6. Synthetic model consists of a medium of the two layers and a block embedded in the second layer. The dimension of the block is $6 \times 2$.

The pseudo section (apparent resistivity) of the forward
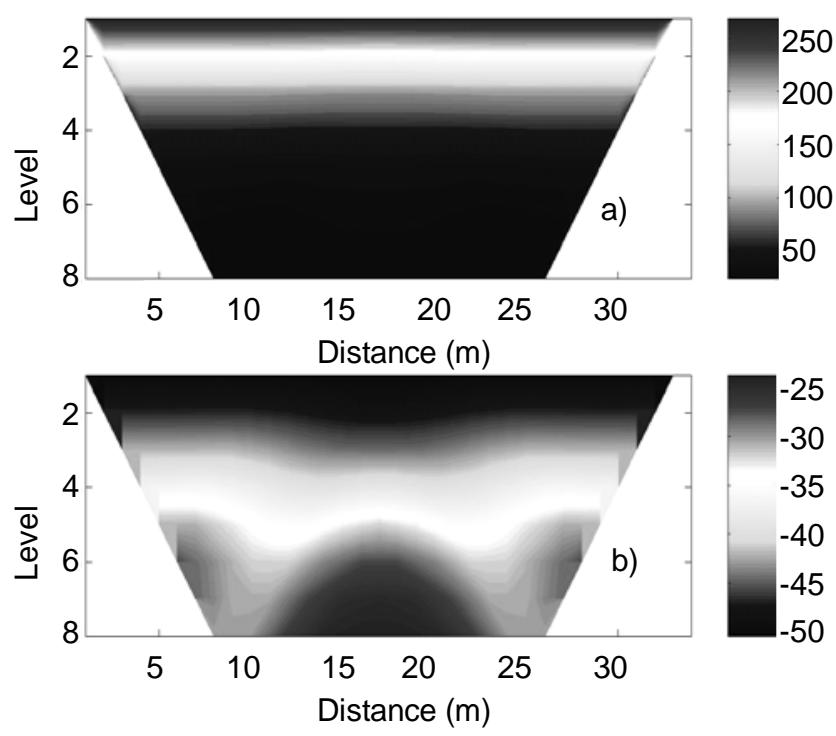

modeling in the frequency of $64 \mathrm{~Hz}$ is showed in Figure 7(a) and (b). We can observe that the block (contamination of the second layer) was not detected by the response of the apparent complex resistivity data. Figure 7(c) presents the apparent fractal parameter $(\eta)$ recovered from the apparent complex resistivity in all frequencies tested in the present study. The presence of the buried body in the second layer was detected and the other parameters had the same behavior of the apparent complex resistivity of the pseudo section in the amplitude and phase angle presented in Figure 7(a) and (b).

The inverse model (amplitude and phase angle) was obtained for the frequencies of 0.1 and $64 \mathrm{~Hz}$ as shown in Figure 8. It can be observed that the response in amplitude has the behavior of the layered medium and the phase angle response detect the contamination (block) of the second layer using low frequency signals. However, the format of the body cannot be very well defined. When the frequency of $64 \mathrm{~Hz}$ is used, only two-layers can be detected.

Figure 9 shows the spatial distribution of the intrinsic fractal model parameters recovered from the inversed data. The block in the second layer was observed more clearly in the image of the parameters $\eta, \delta_{r}, m$ and $\tau_{f}$ than in the inverse model (Figure 8).

The results obtained in the present study were expected on account of the fact that the fractal exponent dominates the phase response mainly in low frequency signals (Rocha, 1995; Farias and Rocha, 2003; Farias, 2004). This is a very important result since in low frequency the fractal parameters carry information about porous roughness of the material. Thus, from field data and with an appropriated inversion algorithm

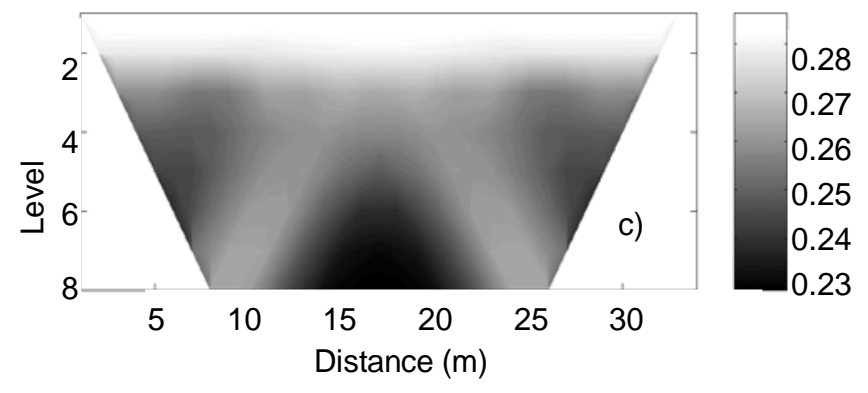

Figure 7. (a) and (b) Apparent complex resistivity Pseudosection in frequency of $0.1 \mathrm{~Hz}$ in amplitude ( $\Omega . \mathrm{m})$ and phase angle (mrad), respectively; (c) apparent fractal parameter $(\eta)$ pseudo section recovered from the apparent complex resistivity pseudo section. 
a)
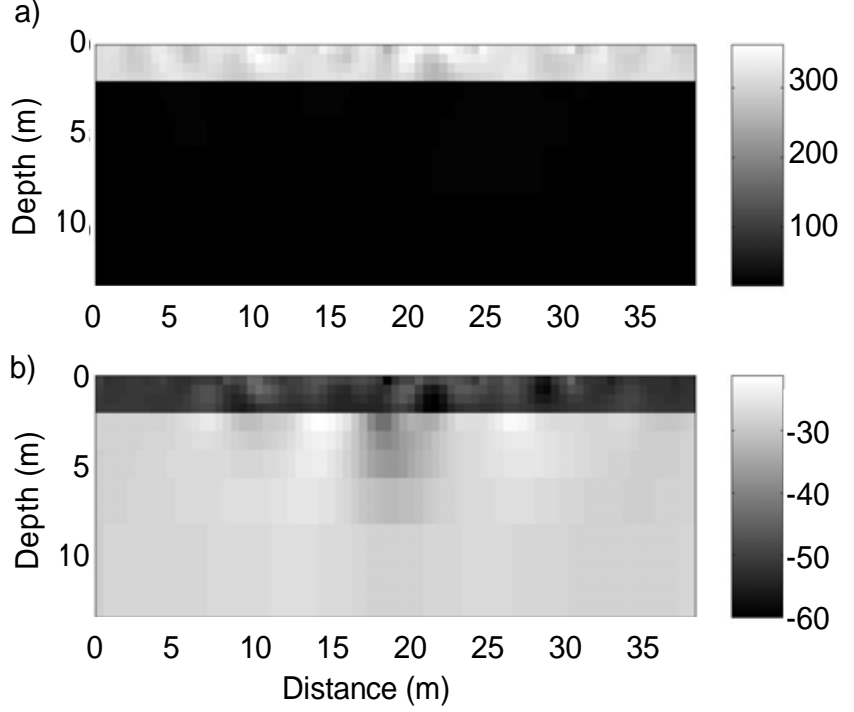

c)
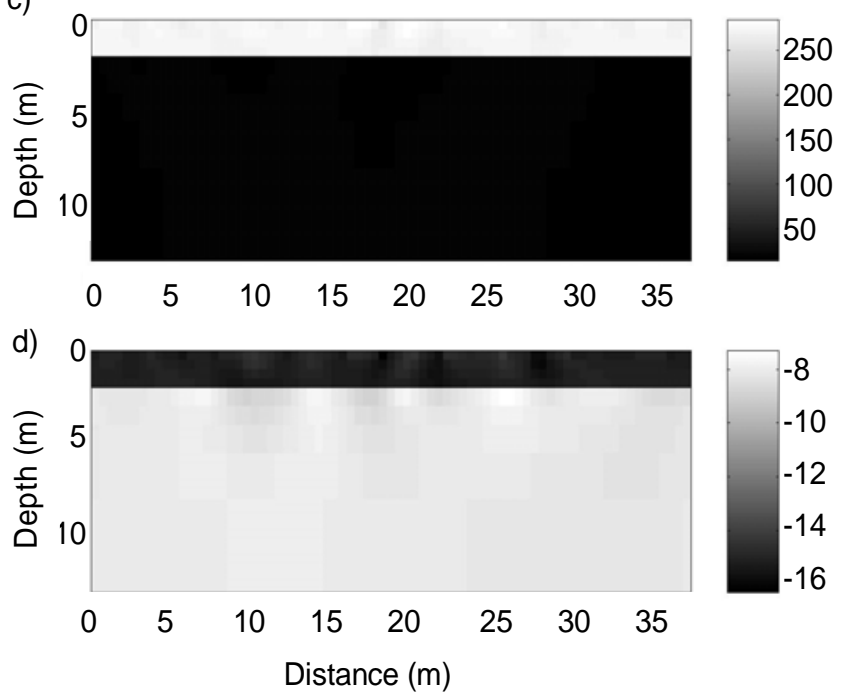

Figure 8. Inverse model of model 2: (a) and (b) amplitude and phase angle in the Frequency $0.1 \mathrm{~Hz}$, respectively; (c) and (d) amplitude and phase angle at the frequency of $64 \mathrm{~Hz}$.
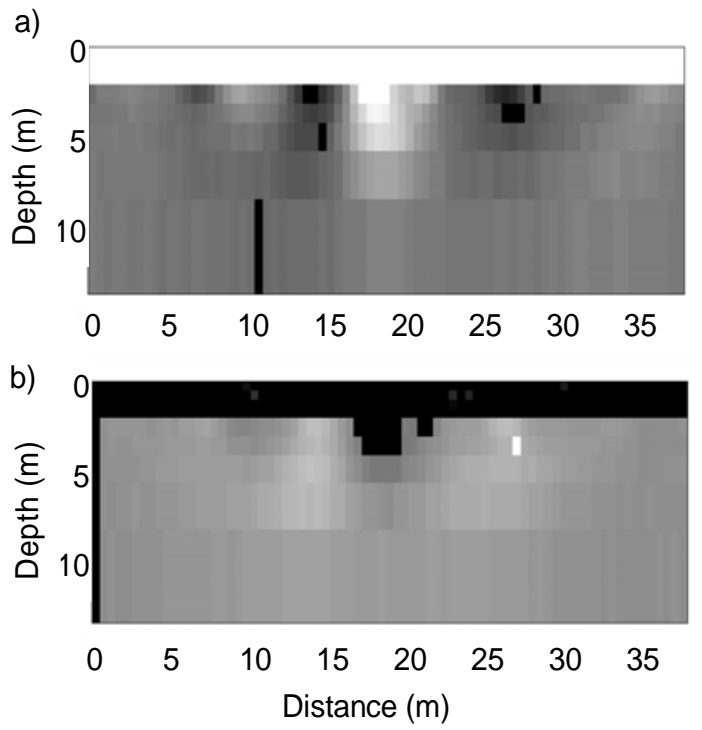
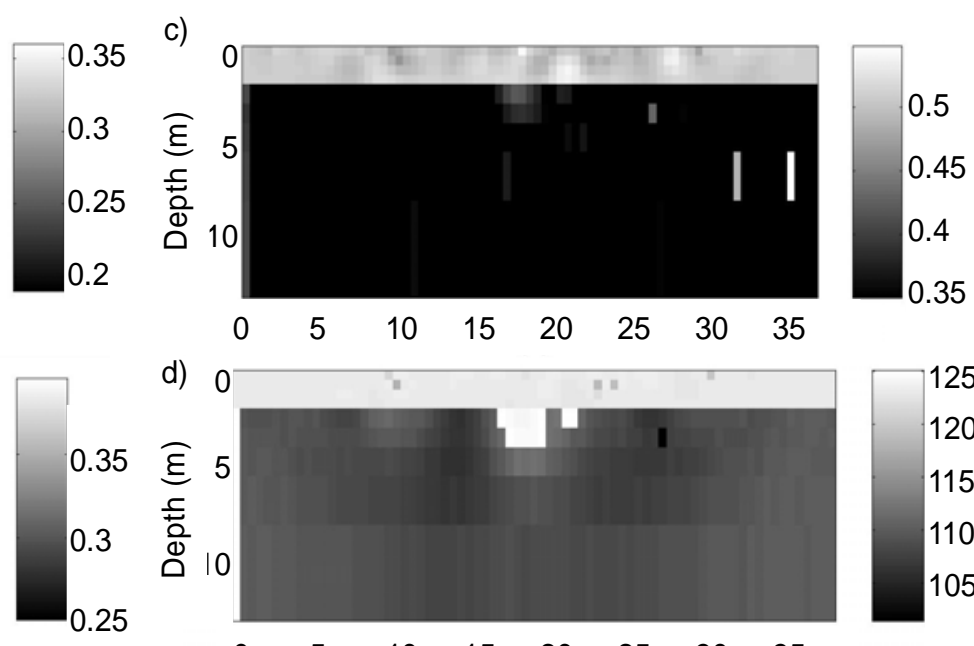

d)

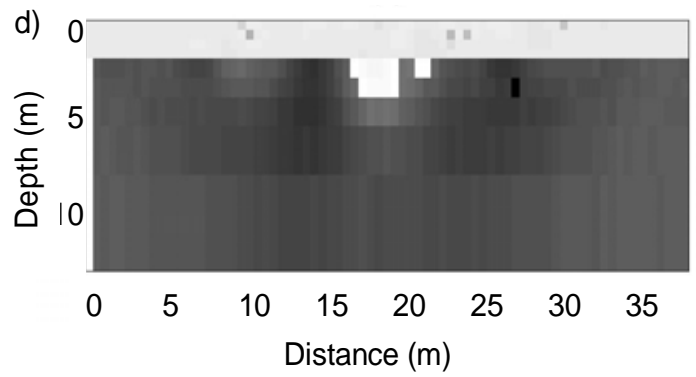

1250 1200 1150 1100 1050

Figure 9. Image of the intrinsic fractal model parameters to model 2: (a) fractal parameter $(\eta)$; (b) parameter $\left(\delta_{\mathrm{r}}\right)$; (c) chargeability $(\mathrm{m})$; (d) fractal relaxation time parameter $\left(\tau_{\mathrm{f}}\right)$. 